\title{
EL FINANCIAMIENTO DE LA INTEGRACIÓN INFRAESTRUCTURAL SUDAMERICANA: LAS DIFICULTADES INSTITUCIONALES DE CONSTITUCIÓN DE UN NUEVO ARREGLO FINANCIERO REGIONAL
}

\author{
Aline Contti Castro' \\ Fernanda $\mathrm{Cimini}^{2}$
}

\begin{abstract}
Este artículo analiza el arreglo de financiamiento de la Iniciativa para la Integración de la Infraestructura Regional Suramericana (IIRSA), buscando identificar cuáles fueron las organizaciones financieras más relevantes y los principales desafíos institucionales enfrentados para la captación de recursos en la región. Teniendo como objeto de análisis las agendas de proyectos prioritarios en el periodo entre 2005-2018, se argumenta que la idea de un arreglo de financiamiento innovador ha fracasado por cuenta de: la capacidad limitada de la inversión pública y la baja capacidad crediticia internacional de la mayoría de los Estados de la región; la permanencia de un ciclo vicioso en que proyectos con recursos ya asignados son los que atraen más recursos; la pequeña contribución de las instituciones multilaterales de desarrollo; la baja participación del sector privado; y la ausencia de liderazgo y de soluciones colectivas, intensificado por las crecientes divergencias políticas entre los gobiernos de la región. Muchas de estas dificultades son típicas de las economías periféricas, resultando en la continuidad de un bajo volumen (leverage) de inversiones regionales en infraestructura.
\end{abstract}

Palabras clave: IIRSA; financiamiento; infraestructura; América del Sur; integración regional.

\section{O FINANCIAMENTO DA INTEGRAÇÃO INFRAESTRUTURAL SUL-AMERICANA: AS DIFICULDADES DE CONSTITUIÇÃO DE UM NOVO ARRANJO FINANCEIRO REGIONAL}

0 artigo analisa o arranjo de financiamento da Iniciativa para a Integração da Infraestrutura Regional Sul-Americana (IIRSA), buscando identificar as instituições financeiras mais relevantes e os principais desafios enfrentados para a captação de recursos na região. Tendo como objeto de análise a carteira de projetos prioritários no período entre 2005 e 2018, argumenta-se que a ideia de um arranjo de financiamento inovador fracassou devido a: limitada capacidade de investimento público na região e baixa capacidade creditícia internacional da maioria dos Estados da região; permanência de um ciclo vicioso em que projetos com recursos já alocados são aqueles que atraem mais recursos; baixa contribuição das instituições multilaterais de desenvolvimento; baixa participação do setor privado; ausência de liderança e soluções para problemas coletivos, intensificados por divergências políticas entre os governos da região. Muitas dessas dificuldades são características das economias periféricas, resultando na continuidade de um baixo volume (alavancagem) de investimentos regionais em infraestrutura.

Palavras-chave: IIRSA; financiamento; infraestrutura; América do Sul; integração regional.

\footnotetext{
1. Doctora en Relaciones Internacionales por la Universidad de Brasilia (IREL-UnB) en colaboración con el Instituto de Ciencias Sociales y Políticas de la Universidad de Lisboa (ISCSP-UL). Docente del Departamento de Relaciones Internacionales y del Programa de Posgrado en Gestión Pública y Cooperación Internacional (PGPCI) de la Universidad Federal de Paraíba (UFPB), Brasil. E-mail: <castroalinec@gmail.com>. Orcid: <https://orcid.org/0000-0002-3595-2445>. 2. Doctora en Sociologia por la Universidad Federal del Rio de Janeiro. Docente del Centro de Desarrollo y Planificación Regional (Cedeplar) de la Universidad Federal de Minas Gerais (UFMG). E-mail: <fcimini@cedeplar.ufmg.br>. Orcid: <https://orcid.org/0000-0003-2170-6443>.
} 


\title{
THE FINANCING OF SOUTH AMERICAN INFRASTRUCTURE INTEGRATION: THE DIFFICULTIES OF SETTING UP A NEW REGIONAL FINANCIAL ARRANGEMENT
}

\begin{abstract}
The article analyses the Initiative for the Integration of the Regional Infrastructure of South America (IIRSA) funding arrangement, aiming to identify the most relevant financial institutions and the main challenges faced in raising funds in the region. Focusing on the agendas of priority projects in the period between 2005-2018, it argues that the idea of an innovative financing arrangement has been undermined by the limited capacity of public investment and access to international credit from most States in the region; the permanence of a vicious cycle in which projects with resources already allocated are the ones that attract more resources; the small contributions from multilateral development institutions; the low level of participation of the private sector; the lack of leadership and collective solutions, which are intensified by political differences among the region's governments. Many of these difficulties are characteristic of peripheral economies, resulting in the continuity of a low volume (leverage) of regional investments in infrastructure.
\end{abstract}

Keywords: IIRSA; financing; infrastructure; South America; regional integration.

JEL: F55; N96; P48; F02.

DOI: http://dx.doi.org/10.38116/rtm23art5

Data de envio do artigo: 16/6/2020; Data de aceite: 24/8/2020.

\section{INTRODUCCIÓN}

Los fenómenos de regionalización son centrales en la vida internacional contemporánea. La necesidad de perfeccionar la integración económica condujo al debate sobre las formas de intensificar el comercio intrarregional, sobre todo con relación a las cuestiones logísticas y de infraestructura física. En ese contexto, en 2000, bajo el liderazgo del presidente brasileño Fernando Henrique Cardoso, fue lanzada la Iniciativa para la Integración de la Infraestructura Regional Sudamericana (IIRSA), con el objetivo de fomentar proyectos compartidos en las áreas de transporte, energía y telecomunicaciones.

Ocho años después, el establecimiento de la Unión de Naciones Suramericanas (Unasur), en 2008, fue uno de los grandes marcos del regionalismo suramericano del siglo XXI, con el rescate de las perspectivas de autonomía y desarrollo regional, tradicionales en el pensamiento integracionista latinoamericano. IIRSA fue entonces incorporada a esta institución, más específicamente al Consejo Sudamericano de Infraestructura y Planificación (Cosiplan).

En términos de financiamiento, en un primer momento, los países recurrieron a las principales instituciones de desarrollo regionales, en especial Banco Interamericano de Desarrollo (BID), Corporación Andina de Fomento (CAF) y Fondo Financiero para el Desarrollo de la Cuenca del Plata (FonPlata) que actuaron como coordinadores de la iniciativa desde su lanzamiento. Además, se crearon nuevas instituciones multilaterales de fomento, como el Banco del Sur y el Banco del Alianza Bolivariana para los Pueblos de Nuestra América (Alba), 
bajo el ambicioso proyecto de una Nueva Arquitectura Financiera Regional (NAFR). Se buscó formar un esquema de financiamiento innovador, de base regional, y reducir la influencia de instituciones que históricamente tuvieron gran poder en la región, como el Banco Mundial y el Fondo Monetario Internacional (FMI). Sin embargo, la actuación de las instituciones regionales de desarrollo quedó abajo de las expectativas. Además, la actual crisis de Unasur impone serios retos al avance de iniciativas de financiamiento en la región.

Teniendo en cuenta ese escenario, este artículo analiza la financiación de los proyectos de integración en el ámbito de IIRSA. Para ello, se busca identificar las propuestas iniciales y el papel de las instituciones financieras más relevantes, teniendo como cuestión central el análisis de los factores institucionales que obstaculizaron la constitución de un arreglo financiero innovador en la región. Este trabajo contribuye a la literatura sudamericana sobre el financiamiento de proyectos de infraestructura regional a través de un análisis económico y político específico del área de relaciones internacionales, fortaleciendo el conocimiento y la transparencia sobre estos proyectos e instituciones, dimensionando el gasto público y la participación privada.

La sección 2 presenta los marcos históricos fundamentales de la integración infraestructural suramericana, con destaque para la formación de las carteras de proyectos y agendas prioritarias. La sección 3 trata de la evolución del debate sobre financiación de proyectos de integración en la región. La sección 4 trae el análisis descriptivo de los proyectos prioritarios de infraestructura de integración, discutiendo la participación de las Instituciones Multilaterales de Desarrollo (IMD) y otros actores en el arreglo de financiamiento de la agenda prioritaria. Finalmente, la sección 5 presenta las principales conclusiones del trabajo.

\section{IIRSA: OBJETIVOS, CARTERA DE PROYECTOS Y AGENDAS PRIORITARIAS}

En el lanzamiento de IIRSA, en 2000, se difundió su Plan de Acción, fruto de la Reunión de Ministros de los Transportes, Telecomunicaciones y Energía de la América del Sur y estableció sus estándares iniciales en términos de: objetivos, acciones básicas y diseño institucional; así como se inauguró el debate sobre los Procesos Sectoriales de Integración (PSI) y los Ejes de Integración y Desarrollo (EID).

El Plan de Acción comienza enunciando la búsqueda de un estándar de crecimiento sustentable basado en el aumento de la productividad y de la competitividad, "que se deriva de procesos productivos basados en la tecnología y el conocimiento, y cada vez menos en la dependencia de la explotación de recursos naturales" (IIRSA, 2000, p. 2). El desarrollo de la infraestructura regional debería realizarse con base en estos marcos, así como promover el desarrollo sostenible a nivel local. En concreto, el plan inicial era mejorar la infraestructura de transporte 
(vial-carretera, portuaria, aeroportuaria, fluvial) y mejorar los procesos logísticos: "sistemas y regulaciones aduaneras, telecomunicaciones, tecnología de la información, mercados de servicios de logística (fletes, seguros, almacenamiento y procesamiento de licencias, entre otros)" (op. cit.).

Nueve acciones básicas fueron previstas en el Plan de Acción: i) diseñar una visión integral de la infraestructura - con el desarrollo sinérgico de las áreas de transporte, energía y telecomunicaciones (contemplando adicionalmente su interrelación con la infraestructura social, ambiental y relativa a la tecnología de la información); ii) identificar ejes de integración y desarrollo para usar como base de una planificación estratégica; iii) modernizar y actualizar los marcos regulatorios e institucionales nacionales relativos a la infraestructura; iv) armonizar las políticas, planes y marcos regulatorios e institucionales entre los Estados; $v$ ) valorizar la dimensión social y ambiental de los proyectos; vi) mejorar la calidad de vida y las oportunidades de la población local en los ejes de integración regional; vii) incorporar mecanismos de participación y consulta; viii) desarrollar nuevos mecanismos regionales para la gestión y financiación compartida de los proyectos, a partir de la actuación del BID, CAF y FonPlata; y $i x$ ) estructurar esquemas financieros innovadores.

Con relación a esta última acción, estaba claro cierta tendencia privatista. El documento establece que los gobiernos e instituciones financieras multilaterales estimulen el capital privado, "a las características y preferencias de los mercados de capitales, al financiamiento de proyectos que constituyan oportunidades comerciales, y que provean una adecuada distribución de riesgos y beneficios entre los sectores público y privado participantes" (IIRSA, 2000, p. 5). En síntesis, uno de los fundamentos económicos centrales de la iniciativa era fomentar la participación privada y desarrollar estructuras financieras innovadores (Moreira, 2006).

La acción más destacada de esta primera fase fue la organización de la Cartera de Proyectos IIRSA, basada en una Metodología de Planificación Territorial Indicativa, con la estructuración de los EID. Con base en estos, se definieron grupos de proyectos, cada uno con un proyecto ancla, de acuerdo con la experiencia brasileña en el plano plurianual de 1996-1999, cuando la propuesta de planificación territorial victoriosa siguió la lógica de corredores de exportación (Couto, 2012).

Se definieron diez ejes. El Eje Mercado Común del Sur (Mercosur)-Chile (entre São Paulo-Buenos Aires-Santiago) es el de mayor peso económico y concentra más del $50 \%$ de la población sudamericana; los Ejes de Capricornio y el Eje Amazonas presentan una fuerte pretensión de conexión bioceánica (y también sirven al flujo de la soja brasileña); el Eje Perú-Brasil-Bolivia, uno de los más pobres, representa una pequeña parte de la economía sudamericana y presentaba bajísima disponibilidad infraestructural al inicio de la Iniciativa, siendo también importante 
en el flujo de granos brasileños. El Eje del Escudo Guyanés representa una lógica de integración sur-norte, entre Roraima y Amazonas (Brasil) y Venezuela, Guyana, Surinam y Guayana Francesa; el Eje del Sur evidencia la búsqueda de una fuerte conexión interoceánica entre Argentina y Chile; el Eje Interoceánico Central representa la propuesta de conectar el Sur-Sureste-Centro Oeste de Brasil a los puertos del Pacífico, en el norte de Chile y sur del Perú; el Eje Hidrovía Paraguay-Paraná es el más reciente, fruto de un recorte más restringido de un proyecto de enlace de vía navegable de la región; y, finalmente, el Eje Andino abarca la costa del pacífico, de Bolivia a Venezuela (Couto, 2012) (figura 1).

FIGURA 1

EID

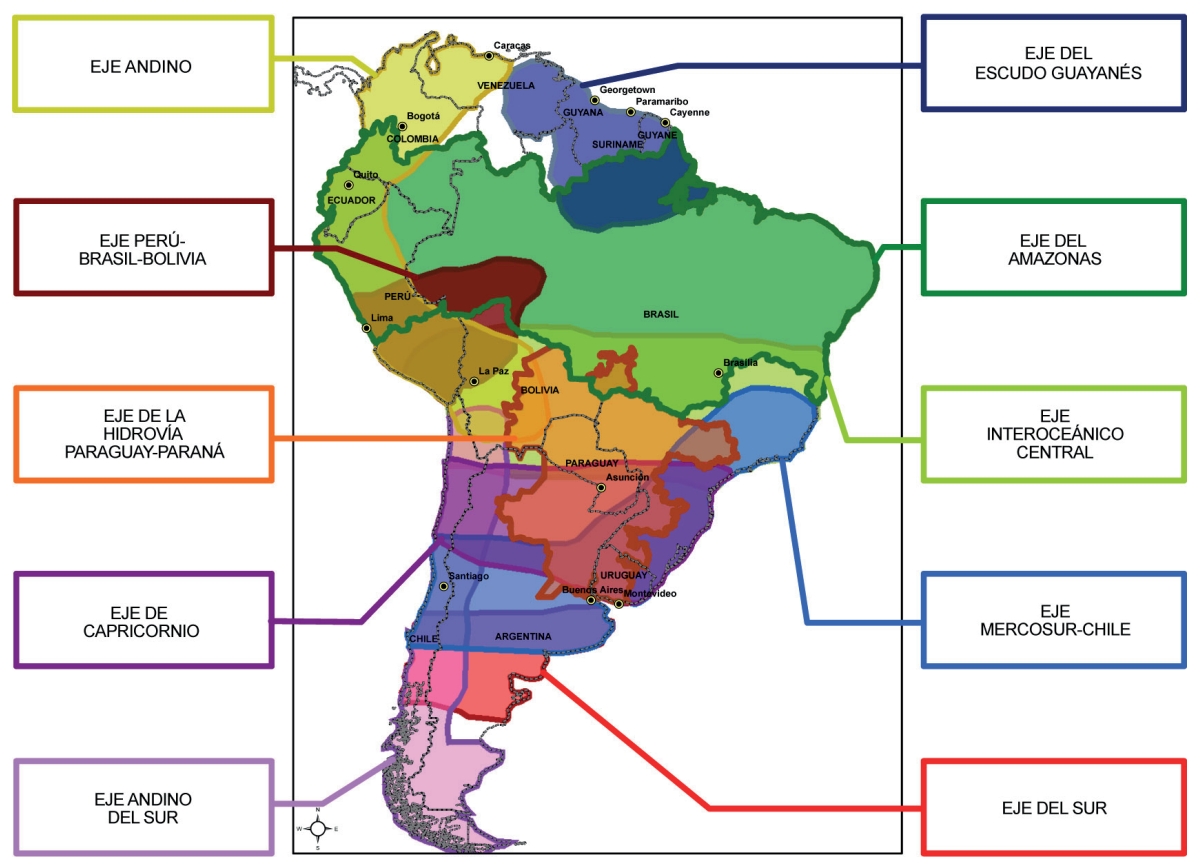

Fuente: IIRSA (2011, p. 66).

Las ideas principales en la organización inicial de la Cartera de Proyectos fueron la definición de grupos de proyectos, sus impactos y su contribución al desarrollo sostenible en términos de sinergias:

la sinergia es vertical cuando la agrupación se da en virtud de las relaciones insumo-producto en una cadena sistémica funcional (por ejemplo, puerto-carretera). Es horizontal cuando se refiere a la utilización de recursos comunes a la facilidad de instrumentación u operación (hidroeléctrica-hidrovía) (IIRSA, 2011, p. 80). 
En cada grupo, el proyecto ancla es el "que confiere sentido a la formación del grupo y viabiliza las sinergias. Este proyecto no es necesariamente el de mayor tamaño, sino lo que constituye el cuello de botella o eslabón faltante de la red de infraestructura" (IIRSA, 2011, p. 81).

En síntesis, los dos principales factores de análisis aplicados en los grupos de proyectos fueron su contribución al desarrollo sostenible, en sus dimensiones económica, social y ambiental; y la cuestión de la factibilidad, con la evaluación de la capacidad de atracción de inversiones y acceso a la financiación de diversas fuentes, públicas y privadas, teniendo en cuenta cuestiones de riesgo y de rentabilidad. Además del financiamiento, otros elementos de factibilidad considerados fueron: la convergencia política y los elementos de viabilidad (marco regulador, demanda, condiciones de ejecución y posibilidad de mitigación de riesgos ambientales) (IIRSA, 2011) (figura 2).

FIGURA 2

Factores de análisis aplicados en los grupos de proyectos

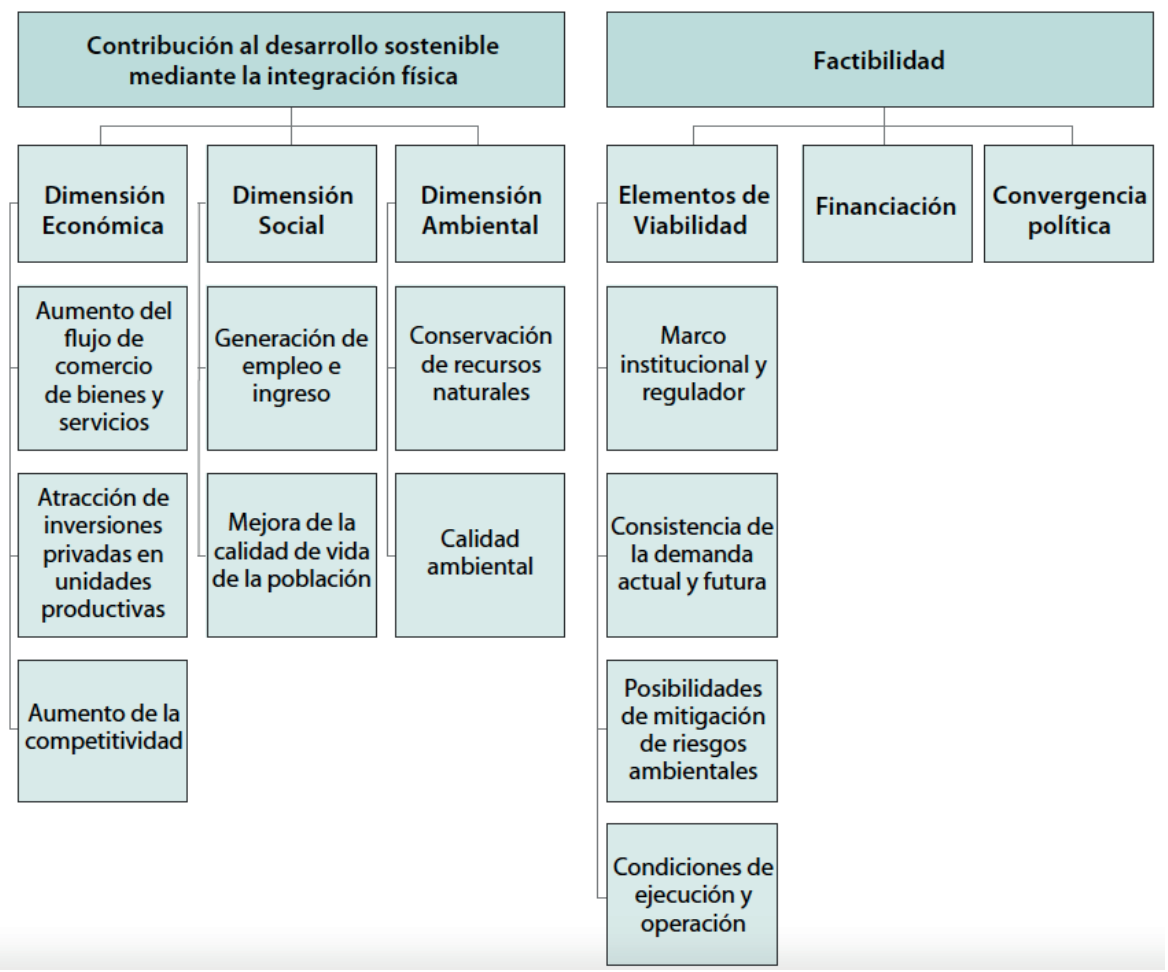


Ante la falta de claridad de los objetivos de la cartera de proyectos, su lentitud, y las dificultades de implementación, los países miembros estimularon la búsqueda por mayor pragmatismo, llevando a la proposición de la Agenda de Implementación Consensuada (AIC) (Costa y Gonzales, 2014). En 2004, se aprobaron los 31 proyectos prioritarios de la AIC para el período 2005-2010 y, en 2005, se definieron los objetivos estratégicos de IIRSA en cuatro líneas de acción principales: i) implementación de la AIC; ii) apoyo a la preparación y financiación de toda la Cartera de Proyectos; iii) mejoramiento cualitativo de la planificación territorial y de la realización de las inversiones; y iv) acciones de divulgación de los avances y logros de la iniciativa (IIRSA, 2011, p. 88).

El documento de evaluación de los diez años iniciales (IIRSA, 2011) resalta el alcance de resultados tangibles, en especial, la conformación de la Cartera de proyectos y la AIC; $y$ de resultados intangibles, como el conocimiento acumulado sobre las oportunidades y restricciones regionales, la cooperación internacional, los desarrollos metodológicos, el capital institucional y la movilización de recursos de la cooperación técnica regional. Sin embargo, se criticaba el hecho de que la Iniciativa no presentaba metas e indicadores específicos para la realización de una evaluación cuantitativa (Costa y Gonzales, 2014). Los objetivos relativos a la armonización de los marcos regulatorios, relativos a los PSI, tampoco avanzaron (Couto, 2012).

Las dificultades de implementación estuvieron relacionadas con factores como la complejidad inherente a una amplia cartera de inversiones en infraestructura; asimetrías económicas y de capacidad institucional entre los países; inexistencia de una estrategia consensuada para expandir la oferta de recursos financieros; falta de mecanismos para reducir los riesgos políticos, regulatorios y financieros; y falta de liderazgo regional para priorizar la integración de la infraestructura física como un elemento fundamental para el desarrollo de la región (Costa y Gonzales, 2015).

Parte de estas dificultades podrían superarse en la segunda fase de la iniciativa, con su incorporación a Unasur.

\subsection{La segunda fase de IIRSA (2011-2017): la incorporación al Cosiplan-Unasur y el Plan de Acción Estratégico}

El Tratado Constitutivo de la Unasur fue celebrado por los doce países de América del Sur en 2008, a partir de las reuniones de los presidentes de la región que se llevaban a cabo desde 2000 y con base en las declaraciones de Cusco (2004, que creó la Comunidad Sudamericana de Naciones), Brasilia (2005) y Cochabamba (2006). Entre los objetivos específicos de la organización están: la integración energética para el aprovechamiento integral, sustentable y solidario de los recursos 
de la región; el desarrollo de una infraestructura para la interconexión de la región y de nuestros pueblos de acuerdo con criterios de desarrollo social y económico sostenibles; y la integración financiera - "mediante la adopción de mecanismos compatibles con las políticas económicas y fiscales de los Estados miembros" (Unasul, 2008, p. 3). ${ }^{3}$

La organización es considerada un marco importante del regionalismo post-liberal que, según Sanahuja (2012), presenta las siguientes características: retorno del énfasis estatal en la conducción de dinámicas de desarrollo; autonomía regional en relación a los intereses políticos norteamericanos; el establecimiento de una agenda positiva, no comercial, con la creación de instituciones y políticas comunes; el establecimiento de nuevos mecanismos de cooperación; y el énfasis en los temas y en la participación social.

La IIRSA fue entonces incorporada al Cosiplan y, con respecto al financiamiento, hubo un cambio significativo de la tendencia privatista inicial (asociada con el regionalismo abierto) a un mayor enfoque en el sector público, siguiendo la tendencia política del regionalismo post-liberal, también llamado regionalismo post-hegemónico (Riggirozzi y Tussie, 2012) o regionalismo desarrollista (Santos, 2016).

En este contexto de cambio institucional, se redefinió la agenda prioritaria, pero la idea política central seguía siendo la misma, lograr, mediante un esfuerzo colectivo, viabilizar el financiamiento de los proyectos de la Agenda de Proyectos Prioritarios de Integración (API). La API, con 77 proyectos, fue aprobada en 2011 y ratificada por los presidentes en 2012. Así, uno de los objetivos establecidos por el Cosiplan era: "Identificar e impulsar la ejecución de proyectos prioritarios para la integración y evaluar alternativas para su financiamiento" (IIRSA, 2012, p. 5).

El Cosiplan elaboró el Plan de Acción Estratégico (PAE) para el período 2012-2022, ratificado en la Sexta Reunión del Consejo de Jefes de Estado de la Unasur, en Lima, con base en los avances anteriores de IIRSA, así como en sus objetivos institucionales y en los objetivos generales de la Unasur.

Los siguientes objetivos específicos del Cosiplan fueron definidos: promover la conectividad de la región a través de la construcción de redes de infraestructura física (de acuerdo con criterios similares a los anteriores, de desarrollo socioeconómico sostenible); diseñar estrategias de planificación; consolidar la cartera de proyectos; fomentar el uso intensivo de las tecnologías de la información y la comunicación; e impulsar la aplicación de metodologías y desarrollo de procesos sectoriales que viabilicen los proyectos de integración física. A partir de eso, el PAE estableció diversas acciones.

3. Las normativas de la Unasur deberían aprobarse por consenso, lo que puede considerarse un elemento de fortalecimiento inicial de la organización, pero representó un componente de dificultades posteriores. 
En términos de financiación, el PAE expresó claramente que "tomando como base la experiencia acumulada en el marco de IIRSA, la cuestión financiera es identificada como una de las principales trabas para la ejecución de los proyectos" (IIRSA, 2012, p. 83). De hecho, hasta 2017, solamente 24\% de la agenda IIRSA fue ejecutada. Tales perspectivas pueden ser observadas por medio de análisis de las fuentes de financiamiento de los proyectos, en especial el contraste entre las inversiones estimadas y ejecutadas en el total de la cartera IIRSA (tabla 1). La mayor parte de los proyectos y de las inversiones ejecutadas fueron financiadas por el sector público (64\%). Las inversiones privadas representaron cerca de 1/4 de las inversiones (US\$11.459 millones). Las IMD financiaron cerca de 6\% de la agenda, siendo que el BID y la CAF tuvieron una participación reducida (alrededor de US\$ 1.100 millones cada uno). El Fondo para la Convergencia Estructural del Mercosur (Focem), la Unión Europea, el Banco Mundial y el Banco Nacional de Desenvolvimento Econômico e Social (BNDES) también participaron en la iniciativa, con pequeñas inversiones relativas (respectivamente US\$ 419, US\$ 75, US\$ 68 y US\$ 67 millones).

TABLA 1

Fuentes de financiación total de los proyectos IIRSA (En US\$ millones)

\begin{tabular}{|c|c|c|c|c|}
\hline Fuente de financiamiento & Número de proyectos & Inversión estimada & Inversión ejecutada & $\%$ ejecutado \\
\hline Sector público & 353 & $83.989,3$ & $30.735,2$ & 37 \\
\hline Participación en el total (\%) & & 42 & 63 & \\
\hline Sector privado & 90 & $43.717,9$ & $11.459,2$ & 26 \\
\hline Participación en el total (\%) & & 22 & 24 & \\
\hline IMD & 96 & $11.794,6$ & $2.996,1$ & 25 \\
\hline Participación en el total (\%) & & 6 & 6 & \\
\hline BID & 36 & $5.716,6$ & 1.211 & \\
\hline CAF & 32 & $4.265,1$ & $1.154,8$ & \\
\hline Focem & 10 & 819 & 419,3 & \\
\hline FonPlata & 6 & 298,9 & 0 & \\
\hline Unión Europea & 4 & 179,5 & 75,3 & \\
\hline Banco Mundial & 3 & 172,5 & 68,5 & \\
\hline $\begin{array}{l}\text { Japan Bank for International } \\
\text { Cooperation (JBIC) }\end{array}$ & 3 & 185,9 & 0 & \\
\hline BNDES & 2 & 157,1 & 67,2 & \\
\hline Otros & 116 & $59.399,8$ & 3.306 & 6 \\
\hline Participación en el total (\%) & & 30 & 7 & \\
\hline Total & 655 & $198.901,6$ & $48.496,5$ & 24 \\
\hline
\end{tabular}

Fuente: IIRSA (2017a, p. 51).

Elaboración de las autoras. 
Así, para superar esa traba, el Cosiplan debería promover en las instancias correspondientes "el diseño de ingenierías financieras adaptadas a los emprendimientos de la Agenda de Proyectos Prioritarios" (IIRSA, 2012, p. 13). La sección siguiente presenta el debate sobre la financiación de esta agenda prioritaria y las tentativas de se crear un arreglo financiero innovador.

\section{EL DEBATE SOBRE LA FINANCIACIÓN DE LOS PROYECTOS IIRSA}

La escasez de capital para inversión de largo plazo es un problema histórico en América Latina (Prebisch, 1962; Furtado, 1970; Bulmer-Thomas, 2003; Perroti y Sánchez, 2011). A pesar de la enorme variedad entre países de la región, la trayectoria reciente no es alentadora. La inversión en infraestructura como porcentaje del producto interno bruto (PIB) ha declinado de 3,7\% en los años 1980 para $2 \%$ en los años 2007-2008, si bien que los sectores público y privado ${ }^{4}$ tuvieron comportamiento distinto, como revela los datos de Comisión Económica para América Latina y el Caribe (Cepal).

TABLA 2

Inversión en infraestructura como porcentaje del PIB

(En \%)

\begin{tabular}{lcccc}
\hline Países seleccionados & $1980-1985$ & $1996-2001$ & $2002-2006$ & $2007-2008$ \\
\hline Sector público & 3,1 & 0,8 & 0,6 & 0,7 \\
Sector privado & 0,6 & 1,4 & 0,9 & 1,3 \\
Total & 3,7 & $\mathbf{2 , 2}$ & $\mathbf{1 , 5}$ & $\mathbf{2 , 0}$ \\
\hline
\end{tabular}

Fuente: Perroti y Sánchez (2011, p. 22).

Si hay desafíos demasiados grandes en el contexto nacional, más grandes lo son todavía en el contexto transnacional. Por ello, el planeamiento para la integración física de la región no puede caminar sin un plan de inversión a largo plazo.

En 2002, un estudio encargado por el BID señalaba los retos que debían ser afrontados por los países latinoamericanos y las alternativas para la financiación de los proyectos IIRSA. El estudio recomendaba que se constituyera una "Autoridad Concedente" que actuaría como agencia ejecutora de los proyectos bajo la forma de un consorcio público. El intento tutelado por esa propuesta pretendía superar la baja institucionalización de cooperación entre los países de la región y reducir los riesgos políticos y legales asociados a los proyectos transnacionales. Esa "Autoridad Concedente" actuaría como una "caja única" para la captación de financiamiento y ejecución de los proyectos. Otra propuesta asociada a esta

4. Está más allá del alcance de este trabajo discutir la posible complementariedad o competencia entre la inversión pública y privada en infraestructura, así como las razones que llevan el sector privado a ampliar sus inversiones. 
iniciativa era la de monetizar las externalidades negativas y positivas de los proyectos, así que cada estado debería aportar fondos de manera proporcional a las externalidades positivas obtenidas por el proyecto.

Más allá de la creación de una "Autoridad Concedente", el estudio recomendaba la adopción de arreglos no tradicionales de financiación. Como la mayoría de los países no disfrutaban de ningún grado de inversión (excepto Chile), la solución propuesta era la creación de una "sociedad de vehículo especial", cuya única función sería la captación de fondos en los mercados financieros a través de préstamos, bonos u otros instrumentos similares (Grupo Analistas, 2002). Ese mecanismo sería posible por medio del unbundling, que consiste en la separación de la financiación de un proyecto y su implementación. Los bancos multilaterales de desarrollo (BMD), como el BID y la CAF, participarían en esa sociedad para aumentar la calificación crediticia de los proyectos IIRSA. Los BMDs ofrecerían tanto préstamos a los gobiernos elegibles a través de cofinanciación, como garantías adicionales a los bonos emitidos por la sociedad. Además, las instituciones multilaterales serían responsables por el acompañamiento técnico de los proyectos y la supervisión de los contratos.

Ese arreglo tendría como objeto hacer la iniciativa IIRSA más atractiva a los inversores privados. Según el estudio, los proyectos transnacionales IIRSA tenían potencial para generar notables beneficios al capital privado, aunque estos serían difusos y distribuidos de forma desigual. Pero la estructura tradicional de financiación privada en infraestructura, que consiste en la fórmula de concesión de responsabilidad por el proyecto al sector privado, no sería suficiente para manejar la complejidad de los proyectos transnacionales. Por ejemplo, los riesgos de cambio en el pago de peajes en carreteras transnacionales exigen medidas para hacer frente a las severas devaluaciones o crisis financieras que pueden ocurrir en uno o más países involucrados. En casos así, los participantes de la sociedad especial ofrecerían garantías para proteger a los proyectos de las fluctuaciones monetarias.

Las propuestas del estudio fueron objeto de discusión entre los representantes de los países en el "Grupo de Trabajo Técnico de Financiamiento" de IIRSA, aunque han sido solo parcialmente incorporadas al plan de trabajo elaborado en la VI Reunión del Comité de Dirección Ejecutiva de IIRSA de 2005. Entre los puntos señalados en el plan de trabajo estaba el reconocimiento de que las exigencias de infraestructura no pueden ejecutarse únicamente con los mecanismos tradicionales de financiación y deben contar con mecanismos innovadores, tales como esquemas de asociación público-privada con garantías, bonos, fondos fiduciarios y otras formas de obtener ingresos, incluso, peajes e impuestos vinculados. Asimismo, el plan reconocía la necesidad de adopción de metodologías de formulación y evaluación de proyectos que permitiesen el análisis del efecto de cada proyecto sobre los futuros ingresos. 
No obstante, el plan de trabajo no mencionaba la creación de ninguna autoridad de coordinación, ni siquiera de una caja única para actuar como fondo común a los proyectos. El plan tampoco destacaba la participación del sector privado o de los bancos multilaterales en la formación de un arreglo de financiación transnacional. Al contrario, la premisa del plan era la de que "diferentes países y diferentes proyectos en un mismo país pueden exigir soluciones diferentes" (IIRSA, 2004, p. 2). Es decir, el plan sugería que cada proyecto debería tener un arreglo propio de financiación, dispensando la creación de una institución intergubernamental. A partir de ahí, los debates sobre arreglos de financiación transnacional no llegaron a un resultado sólido. El Informe de Evaluación de la AIC (IIRSA, 2010) expresa el hecho de que la realización de una "Marca IIRSA" para facilitar la financiación de los proyectos en las instituciones multilaterales no ocurrió y así se debilitaron los debates sobre financiamiento. "Es verificable que, a partir de 2005, la iniciativa no gastó más energía en la discusión de modelos genéricos con respecto al financiamiento y se pasó a buscar soluciones proyecto por proyecto". (op. cit., p. 15).

La falta de financiamiento siguió siendo un reto para la agenda de proyectos de integración y la iniciativa no logró crear nuevos mecanismos de financiación. Un modesto destaque fue la creación del fondo del BID (de US\$20 millones) para la elaboración de estudios iniciales (IIRSA, 2011). Se ha alegado que el tratamiento de los bancos multilaterales involucrados en la iniciativa a los proyectos de la cartera IIRSA no podría diferenciarse de lo que se ofrecía a los otros socios institucionales (Couto, 2012).

En 2012, el tema volvió a ser discutido cuando Cosiplan propuso la creación de un "Grupo de Trabajo sobre Mecanismos de Financiamiento y Garantía". La justificación para el grupo era de que la implementación de los proyectos no había alcanzado las expectativas de ejecución y eso fue consecuencia, sobre todo, de la falta de financiamiento. Además, el Cosiplan reconocía que la IIRSA poco había avanzado en las cuestiones relacionadas a la factibilidad financiera de los proyectos. Las primeras propuestas del grupo fueron presentadas el año siguiente, en la IV Reunión Ordinaria del Consejo de Ministros, en 2013. El grupo recomendaba la creación de un programa de financiación regional, la creación de un sistema regional de garantías, la expansión del Comité de Coordinación Técnica (CCT) (con la inclusión de otros bancos de desarrollo como el Banco Chino o el Banco del BRICS) y, sobre todo, la consolidación del Banco del Sur, a través de la expansión del presupuesto reembolsable y no-reembolsable.

Aunque esas propuestas buscasen atraer más inversiones de los BMD, indirectamente resulta que ellas acaban sobrecargando el presupuesto público, dado que gran parte de estos préstamos son reembolsables, es decir, impactan 
la deuda soberana. ${ }^{5}$ Asimismo, el plan presentado en 2013 no contemplaba estrategias de captación privada, ni la creación de modelos transnacionales de financiamiento. Todo indica que las discusiones de modelos genéricos de financiamiento no han sido recuperadas ya que siguió la búsqueda por soluciones individuales de proyecto. De hecho, el documento PAE explica que:

es responsabilidad de los países obtener los recursos necesarios para el financiamiento de los estudios de pre inversión que requieren los proyectos y para su ejecución, pudiendo hacerlo con recursos propios o acudir a los organismos multilaterales de crédito que conforman el CCT, a otros organismos de financiamiento, a entidades de cooperación o a la banca pública o privada (IIRSA, 2017b, p. 58, énfasis añadido).

Además, el plan preveía que:

las Coordinaciones Nacionales de los países miembros realizarán las gestiones institucionales internas que sean necesarias para que los proyectos de la Cartera y de la API sean priorizados e incorporados en los programas de financiamiento que anualmente los países negocian con las entidades multilaterales de financiamiento y de cooperación, a fin de impulsar la ejecución de estos proyectos (IIRSA, 2017b, p. 59).

El análisis de la evolución del debate sugiere que las premisas y propuestas presentadas por el Grupo de Trabajo de Cosiplan para financiar la API siguen similares a las presentadas por el Grupo de Trabajo de IIRSA para AIC: $i$ ) ausencia de un plan global de financiamiento; ii) elevada confianza en la capacidad de los tesoros nacionales para financiar proyectos; iii) ausencia de incentivos para captar la inversión privada; y iv) participación limitada de los BMD, en función de la capacidad de endeudamiento individual de los países.

La manutención de esas premisas tiene serias implicaciones para los rumbos de los proyectos de infraestructura en la región.

Así, las dificultades de la primera fase de la iniciativa no se superaron con su incorporación a Unasur. A los obstáculos anteriores, se sumaron las crecientes diferencias políticas entre Brasil y Venezuela en el área financiera. Véase, por ejemplo, las dificultades de ratificación del Banco del Sur. ${ }^{6}$

Potencialmente, la idea de crear una institución autónoma podría implicar en: evaluaciones de riesgos más favorables que las del mercado internacional; más espacio para los países de menor desarrollo relativo; menos condicionalidades impositivas y restrictivas (de políticas públicas); rating institucional potencialmente mayor que los nacionales; construcción de capacidades supranacionales en la evaluación de proyectos

5. Diferente del Focem que es un fondo no-reembolsable, pero es de dimensión pequeña.

6. El Banco del Sur se constituyó formalmente en 2013, con la ratificación de Argentina, Bolivia, Ecuador, Uruguay y Venezuela, pero aún no ha sido ratificado por Brasil y Paraguay. 
y en la distribución de sus costos y beneficios; apoyo al desarrollo productivo regional de la región de forma ampliada (más allá de las limitaciones nacionales).

Todavía, Brasil no ha ratificado la propuesta del Banco do Sul, se ha opuesto a la creación de nuevas instituciones financieras y al uso de sus reservas nacionales como garantía. En otras palabras, fue en contra de las audaces propuestas de Hugo Chávez, de una nueva arquitectura financiera regional. Este hecho fue expuesto por Celso Amorim (ministro de Relaciones Exteriores de Brasil) en declaraciones públicas, donde reforzó el uso de instituciones existentes, como BNDES, CAF y Focem. ${ }^{7}$ Con relación al Banco do Sul, Santos (2016) se refiere a "objeciones del Banco Central y del Ministerio de Economía [de Brasil] contra el uso de reservas internacionales para financiar inversiones"; así como a la posición brasileña contraria a las propuestas venezolanas sobre el igual peso del voto de los países y los préstamos a "fondos perdidos".

Cabe mencionar que, en el inicio del gobierno Lula da Silva (2003), se destacó cierta desconfianza de la iniciativa, teniendo en vista la presencia del BID en el CCT y la ausencia del BNDES, así como en relación a la "fuerte tendencia privatista de las discusiones relativas a la armonización de los marcos regulatorios regionales, particularmente el de energía" (Couto, 2012, p. 138). Sin embargo, en función de las presiones externas y de los intereses nacionales, IIRSA entró en la agenda de la política exterior brasileña, con el gobierno buscando insertar el BNDES en la iniciativa ${ }^{8}$ y fortalecer así la internacionalización de grandes empresas brasileñas.

La ausencia de liderazgo financiero de Brasil, la mayor potencia regional, se evidenció por la falta de acciones propositivas y de construcción de consensos, contribuyendodecisivamenteal no establecimientodemecanismos definanciamiento coordinados y colectivos. Además, conforme Costa y Gonzales (2015), la falta de liderazgo dificultó mantener el consenso inicial sobre la importancia de integrar la infraestructura física para aumentar la competitividad de la región.

En el caso de Venezuela, Hugo Chávez criticaba abiertamente la iniciativa, la fuerza del BID y de los intereses privados en este ámbito, pasando a ausentarse de las reuniones. Otro tema de difícil conciliación con Brasil fue el sector de energía (Céspedes y Agostini, 2014). La rediscusión del modelo regulatorio energético brasileño estancó la discusión del grupo (Couto, 2012) y se destacaron otras tensiones, como las evidenciadas por la nacionalización de la refinería de Petrobras en Bolivia (en 2006) en el gobierno de Evo Morales, con apoyo del gobierno de Venezuela.

7. Ver, por ejemplo, la entrevista de Celso Amorim sobre el tema, disponible en: <https://tinyurl.com/yyesq8l6> Acceso en: jul. 2020.

8. El banco, en la cartera IIRSA-Cosiplan, aparece como una fuente de financiamiento para solo dos proyectos, ambos en Bolivia (Wegner, 2018). 
Después de este análisis acerca de los debates financieros de la IIRSA, la sección que sigue compara la financiación de las agendas de proyectos prioritarios (AIC y API) en los dos períodos de la iniciativa y apunta los avances y retos de eses arreglos. Se espera que en las agendas prioritarias el porcentaje de ejecución sea más grande y que el arreglo de financiamiento sea más equilibrado ya que contemplan proyectos políticamente más relevantes y económicamente más viables (o, por lo menos, con asignación de recursos garantizada).

\section{EL FINANCIAMIENTO DE LOS PROYECTOS PRIORITARIOS}

La cartera de proyectos de infraestructura de integración (actualmente conocida como Cartera Cosiplan) fue creada en 2004 y desde ahí ha pasado por sucesivas actualizaciones. Hasta 2017, la cartera registraba un total de 562 proyectos con una inversión estimada en US\$ 198.901 millones, los cuales se distribuyen de la siguiente manera: una cartera activa compuesta de 409 proyectos con una inversión estimada en US\$150.405 millones, y 153 proyectos concluidos con una inversión de US\$ 48.496 millones (IIRSA, 2017a).

A lo largo de los últimos trece ańos, la cartera de proyectos prioritarios de infraestructura de integración ha tenido dos importantes hitos: la AIC, que radicaba en 31 proyectos prioritarios considerados relevantes para el proceso de integración física para el periodo entre 2005 y 2010, sobretodo en el sector de transportes; y la API, ya en el marco del Cosiplan, para un horizonte de diez ańos (2012-2022). Así como la AIC, la API también está constituida por proyectos relevantes para la integración física regional y puede ser comprendida como una continuidad de la anterior, aunque bajo un contexto institucional diferenciado. Desde su creación en 2011, la API ha mantenido los 31 proyectos estructurados originales y ha añadido varios otros proyectos individuales. ${ }^{9}$

Los portfolios de la AIC y de la API están compuestos por 31 y 77 proyectos respectivamente, con inversiones estimadas en U\$ 14.023 millones (AIC) y US\$ 23.618 millones (API) (IIRSA, 2011 e SIP ${ }^{10}$ ). En el informe de cierre de la AIC (IIRSA, 2010, p. 41), la evolución de la inversión estimada pasó de US\$ 5.833 millones en 2005 a US\$ 14.023 millones en 2010. Cinco ańos tras su implementación, dos proyectos se encontraban concluidos $(6,5 \%$ del monto estimado), diecinueve en ejecución (61,3\% del monto), ocho en preparación ( $25,8 \%$ del monto) y dos se encontraban todavía en perfil.

Ya en la API, en la evaluación quinquenal de 2017, dieciséis proyectos estaban concluidos (12\% del monto total en inversión estimada), 28 en ejecución

9. Por ejemplo, en 2013, seis proyectos individuales resultaron en el desmembramiento de diecinueve proyectos individuales más específicos.

10. Disponible en: <http://cosiplan.org/proyectos/>. 
(52\% del monto estimado), 27 en preparación (35\% del monto) y tres en perfil. En marzo de 2019, de acuerdo con el Sistema de Información de Proyectos (SIP-IIRSA), los números eran los siguientes (tabla 3).

TABLA 3

Análisis comparada de las carteras de proyectos de AIC y API (En US\$ millones)

\begin{tabular}{lcccccc}
\hline \multirow{2}{*}{ Situación } & \multicolumn{2}{c}{ AIC (2005-2010) } & & \multicolumn{2}{c}{ API (2012-2018) } \\
\cline { 2 - 3 } \cline { 5 - 6 } & Proyectos & Inversión estimada & & Proyectos & Inversión estimada \\
\hline Concluido & 2 & 22 & & 16 & 1.950 \\
Ejecución & 19 & $7.677,4$ & & 29 & 8.729 \\
Preparación & 8 & $6.320,8$ & & 29 & 12.893 \\
Perfil & 2 & 2,8 & & 3 & 46 \\
Total & 31 & $\mathbf{1 4 . 0 2 3 , 0}$ & & 77 & $\mathbf{2 3 . 6 1 9 , 0 0}$ \\
\hline
\end{tabular}

Fuente: IIRSA (2010). Informaciones sobre API extraídas de la base de datos SIP-IIRSA - actualizado para marzo de 2019. Disponible en: <http://cosiplan.org/proyectos/>. Acceso en: mar. 2019.

Elaboración de las autoras.

La comparación entre los primeros cinco ańos de las dos agendas indica un aumento en cuanto al monto concluido y en ejecución. El número superior de proyectos individuales de la API se debió al aumento de la información cada vez más exacta sobre los proyectos a lo largo de los últimos años, ocasionando mayor desagregación de los proyectos. Eso permitió registrar mejor los avances y facilitar el monitoreo (IIRSA, 2017b). ${ }^{11}$

Teniendo presente el conjunto total de proyectos nacionales concluidos y en ejecución de la API (tabla 4), se nota que Perú es el país con mayor participación en la cartera, representando casi la mitad de los costos totales (45\%). A continuación, vienen Colombia y Argentina, con cerca de 15\%. Según Costa y Gonzales (2014), más proyectos fueron financiados en los ejes más consolidados económicamente (en especial el Mercosur-Chile, Interoceánico Central y Capricornio), lo que demuestra el carácter más objetivo de esa agenda en comparación a la AIC.

Otra característica de la API es el alcance transnacional limitado de los proyectos, ya que se observa una fuerte concentración en proyectos nacionales (un 99.5\% de la inversión en proyectos concluidos o en ejecución). Se nota también que los proyectos binacionales o multinacionales son en su mayoría financiados por el sector público (85\%). Eso significa que mientras más grandes sean los riesgo políticos y legales asociados a los proyectos transnacionales, más baja será la participación de otros inversores que no el Estado.

11. Los valores actualizados de la cartera AIC para julio de 2018 son: $24 \%$ concluido, 27\% en ejecución, 49\% en pre-ejecución y $1 \%$ en perfil. Fuente: base de datos de proyectos IIRSA. 
TABLA 4

Distribución del costo total de proyectos API concluidos y en ejecución por país (hasta 2018)

\begin{tabular}{lccc}
\hline \multirow{2}{*}{ Países } & Número de proyectos & \multicolumn{2}{c}{ Costo total de proyectos nacionales } \\
\cline { 3 - 4 } & & US\$ millones & $\%$ \\
\hline Peru & 14 & 6.495 & 45,81 \\
Colômbia & 3 & 2.162 & 15,25 \\
Argentina & 2 & 1.980 & 13,97 \\
Paraguai & 4 & 1.840 & 12,98 \\
Brasil & 6 & 846 & 5,97 \\
Bolívia & 2 & 400 & 2,82 \\
Uruguai & 5 & 377 & 2,66 \\
Chile & 1 & 76 & 0,54 \\
Venezuela & 1 & 2 & 0,01 \\
Total & $\mathbf{3 8}$ & $\mathbf{1 4 . 1 7 7}$ & $\mathbf{1 0 0 , 0 0}$ \\
\hline
\end{tabular}

Fuente: Informaciones sobre API extraídas de la base de datos SIP-IIRSA - actualizado para julio de 2018. Disponible en: $<$ http://cosiplan.org/proyectos/>. Acceso en: jul. 2018.

Elaboración de las autoras.

Obs.: La tabla considera solamente los costos totales de proyectos nacionales.

De hecho, al considerar los tipos de financiamiento de los proyectos concluidos y/o en ejecución de las carteras AIC y API (tabla 5) se observa el aumento significativo del peso del financiamiento exclusivamente público (del 20 al 47\%) y un ligero aumento del financiamiento exclusivamente privado (del 22 al 26\%). En la AIC, aunque las instituciones multilaterales participaron en 9 de los 31 proyectos, no hubo proyectos financiado exclusivamente por ellas, mientras que en la API hay un proyecto con financiación total del BID y uno de CAF, totalizando U\$ $\$ 556$ millones. ${ }^{12}$ Asimismo, se destaca la reducción de los proyectos con financiamiento compartido (del 58 al 22\%).

TABLA 5

Análisis comparada de las fuentes de financiamiento de las carteras AIC y API: proyectos concluidos y en ejecución

\begin{tabular}{lccccc}
\hline \multirow{2}{*}{ Tipo de financiamiento } & \multicolumn{2}{c}{ AIC (2005-2010) } & & \multicolumn{2}{c}{ API (2012-2018) } \\
\cline { 2 - 3 } \cline { 5 - 6 } & US\$ millones & $\%$ & & US\$ millones & \% \\
\hline Público & 2.172 & 20 & & 4.949 & 47 \\
Privado & 2.305 & 22 & & 2.720 & 26 \\
IMD & - & 58 & 566 & 5 \\
Compartido & 6.157 & 100 & & 10.384 & 22 \\
Total & 10.635 & - & & 10.630 & 100 \\
\hline
\end{tabular}

Fuente: Informaciones sobre AIC extraídas de Costa y Gonzalez (2014) a partir de datos disponibles en IIRSA - VI Informe de AIC, jul. 2010. Informaciones sobre API extraídas de la base de datos SIP-IIRSA. Disponible en: <http://cosiplan.org/ proyectos/>. Acceso en: jul. 2018.

Elaboración de las autoras.

12. Proyecto Línea de Transmisión 500 KV (Yacyretá - Villa Hayes) - CAP68 y Doble Vía La Paz - Santa Cruz - IOC80. 
Respecto a los proyectos con financiamiento compartido (tabla 6), se observa la significativa reducción de la participación privada (del 51 al 16\%) ${ }^{13}$ y el aumento de la participación del sector público (del 22 al 51\%). En cuanto a las IMD, se señala la manutención relativa a su participación en proyectos compartidos. Una mirada hacia los proyectos con fuente de financiamiento compartido con las IMD señala una fuerte concentración en los sectores de transporte, siendo cuatro de carreteras, dos de ferrovías y uno proyecto de logística (desaguadero), bien como dos proyectos de energía (líneas de transmisión de Itaipú y Yacyretá). La participación privada está concentrada en dos proyectos de carretera en Perú.

TABLA 6

Análisis comparada de proyectos de las carteras AIC y API con fuente de financiamiento compartido: proyectos concluidos y en ejecución

\begin{tabular}{|c|c|c|c|c|}
\hline \multirow{2}{*}{ Tipo de financiamiento } & \multicolumn{2}{|c|}{ AIC (2005-2010) } & \multicolumn{2}{|c|}{ API (2012-2018) } \\
\hline & US\$ millones & $\%$ & US\$ millones & $\%$ \\
\hline Público & 1.337 & 22 & 1.176 & 51 \\
\hline Privado & 3.165 & 51 & 368 & 16 \\
\hline IMD & 1.655 & 27 & 715 & 31 \\
\hline Indefinido & - & - & 25 & 1 \\
\hline Total & 6.157 & 100 & 2.384 & 100 \\
\hline
\end{tabular}

Fuente: Informaciones sobre AIC extraídas de Costa y Gonzalez (2014) a partir de datos disponibles en IIRSA - VI Informe de AIC, jul. 2010. Informaciones sobre API extraídas de la base de datos SIP-IIRSA en julio de 2018.

Elaboración de las autoras.

Entre las IMD con más participación, gana destaque el Focem, que ha aportado US\$ 533 millones en proyectos concluidos y en ejecución, contra US\$ 360 millones del BID y US\$ 387 millones de la CAF. Cabe señalar que CAF mantuvo su participación relativamente estable mientras que BID ha reducido significativamente del 55 al 28\% (tabla 7).

Sin embargo, cuando se observa la distribución de países en la cartera de proyectos aprobados del BID para América Latina (tabla 8) se nota la importancia de los proyectos regionales, que representaron $28 \%$ de la cartera de los proyectos aprobados entre 2005 y 2017. Es curioso el contraste entre el volumen de inversión aprobado para proyectos regionales, que suma US\$ 3,9 billones, y el volumen aportado en las carteras AIC y API, de US\$ 1,2 billones, lo que demuestra la poca importancia de la iniciativa para el banco. Sin embargo, a pesar del pequeño volumen de inversiones del banco, se destaca su amplio alcance internacional, con acción en casi todos los países de la región.

13. El financiamiento privado en la agenda de la AIC se refiere a dos proyectos en Perú. Los proyectos son los siguientes: i) carretera Paita-Tarapoto-Yurimaguas, puertos y centros logísticos, ubicados en el eje amazónico, por un valor estimado de US\$ 937 millones, de los cuales US\$ 739 millones provienen de fuentes privadas; y ii) autopista Lima-Tingo María-Pucalpa, puertos y centros logísticos, también en el eje amazónico, por US\$2,900 millones, de los cuales US\$2,400 corresponden al sector privado (Costa y Gonzalez, 2015). 
El Financiamiento de la Integración Infraestructural Suramericana: las dificultades institucionales de constitución de un nuevo arreglo financiero regional

TABLA 7

Contribuciones totales de las IMD: proyectos concluidos y en ejecución

\begin{tabular}{lccccc}
\hline \multirow{2}{*}{ Participación de los BMD } & \multicolumn{2}{c}{ AIC (2005-2010) } & & \multicolumn{2}{c}{ API (2012-2018) } \\
\cline { 2 - 3 } \cline { 5 - 6 } BID & US\$ millones & $\%$ & 55 & US\$ millones & \% \\
CAF & 893 & 33 & 360 & 387 & 30 \\
Focem & 536,80 & & 533 & 42 \\
Otros & 0 & 12 & - & - \\
Total & 194 & 100 & 1.281 & 100 \\
\hline
\end{tabular}

Fuente: Informaciones sobre AIC extraídas de Costa y Gonzalez (2014) a partir de datos disponibles en IIRSA - VI Informe de AIC, jul. 2010. Informaciones sobre API extraídas de la base de datos SIP-IIRSA en julio de 2018.

Elaboración de las autoras.

TABLA 8

Inversión aprobada del BID: proyectos concluidos y en ejecución (2005-2017)

\begin{tabular}{|c|c|c|}
\hline País & US\$ millones & Porcentual \\
\hline Regional & 3.935 & 28 \\
\hline Argentina & 3.197 & 22 \\
\hline Brasil & 2.245 & 16 \\
\hline Panamá & 712 & 5 \\
\hline México & 604 & 4 \\
\hline Costa Rica & 552 & 4 \\
\hline Bolivia & 540 & 4 \\
\hline Nicaragua & 404 & 3 \\
\hline Colombia & 300 & 2 \\
\hline Ecuador & 294 & 2 \\
\hline Honduras & 284 & 2 \\
\hline Guatemala & 243 & 2 \\
\hline Chile & 177 & 1 \\
\hline Perú & 162 & 1 \\
\hline Haití & 117 & 1 \\
\hline Suriname & 106 & 1 \\
\hline El Salvador & 103 & 1 \\
\hline República Dominicana & 72 & 1 \\
\hline Paraguay & 69 & 0 \\
\hline Uruguay & 61 & 0 \\
\hline Bahamas & 36 & 0 \\
\hline Belice & 27 & 0 \\
\hline Jamaica & 7 & 0 \\
\hline Barbados & 6 & 0 \\
\hline Guyana & 0 & 0 \\
\hline Trinidad and Tobago & 0 & 0 \\
\hline Total & 14.252 & 100 \\
\hline
\end{tabular}

Fuente: BID. Disponible en: <https://tinyurl.com/y5vo5g7g>. Acceso en: mayo 2019.

Elaboración de las autoras. 
Así, el arreglo de financiamiento de las carteras prioritarias en los periodos analizados no es tan distinto del arreglo general de IIRSA, presentado anteriormente, excepto por el porcentaje de ejecución, que es superior a los $24 \%$. A pesar de haber logrado un mejor desempeño en la ejecución de proyectos en relación con la AIC, la API no ha logrado avanzar en la creación de un arreglo de financiamiento innovador para fomentar proyectos de infraestructura en la región. Al contrario, el volumen de inversiones privadas se mantuvo relativamente estable y la participación de los IMD fue baja. Así pues, los presupuestos públicos asumieron la mayor parte de las inversiones.

En cuanto a la iniciativa privada, Costa y Gonzalez (2015) identificaron al menos tres características comunes en las preferencias privadas por proyectos de la cartera de AIC: i) países con un marco regulatorio favorable a la participación del sector privado en obras públicas, como es el caso de Chile y Perú; ii) preferencia por el modelo de concesión en el sector del transportes; iii) asignación de presupuesto multilateral o público, revelando la importancia del sector público para estimular el capital privado. En general, esas características fueran mantenidas en la API, ya que no hubo un cambio significativo en los sectores apoyados o en las tasas de rendimiento de los proyectos. Aun así, hubo una reducción en la participación privada en proyectos de financiamiento compartido de la API.

Con respecto a las IMD, se destaca la baja participación del BID en el arreglo financiero. A pesar de la participación activa de esa institución en los sectores de transporte y logística, el volumen de la inversión en la cartera de prioridades de IIRSA/Cosiplan es tres veces más pequeña que la agenda del banco para proyectos regionales. Eso indica que la principal limitación, en el caso del BID, no es tanto la capacidad de financiación adicional de esa organización, sino la falta de interés político en la iniciativa. ${ }^{14}$ También se apunta la ausencia de instituciones externas a la región en el arreglo de financiamiento de proyectos prioritarios, como el Banco Mundial y la propia Unión Europea, que financiaron otros proyectos de IIRSA. ${ }^{15}$

\section{CONSIDERACIONES FINALES}

La IIRSA es una audaz iniciativa regional, con previsión y asignación de gran volumen de inversiones infraestructurales, fruto de las primeras reuniones de presidentes en la historia de América del Sur. Por medio de esta, se buscó crear consensos, realizar estudios y avanzar en el diálogo y en la coordinación política. Se constituyó, por lo tanto, como un medio de fortalecimiento y de atención a los intereses regionales. La construcción de un sistema público de información

14. Con respecto a los sectores apoyados por el BID, la mayoría de los proyectos son de infraestructura y apoyo al sector productivo (industria, agricultura, comercio y servicios).

15. Importante señalar que a pesar de la relevancia de las inversiones del Banco Mundial en América del Sur, la estrategia del banco ha cambiado en la última década y infraestructura no pertenece más a la agenda de prioridades para la región. 
(SIP, disponible en el sitio de la organización) también constituye un diferencial con relación a otros proyectos y organizaciones regionales y ha viabilizado la realización de esta investigación. Sin embargo, en otros aspectos poco se avanzó, como en cuestiones de sostenibilidad, participación local, construcción de marcos regulatorios y estructuración de mecanismos de financiación innovadores.

En síntesis, la idea de un arreglo de financiamiento innovador ha fracasado por cuenta de obstáculos típicos de economías periféricas como la capacidad limitada de la inversión pública y la baja capacidad crediticia internacional de la mayoría de los Estados de la región. Otros factores que dificultaron el proceso fueron: la pequeña contribución de las instituciones multilaterales de desarrollo; la baja participación del sector privado; la permanencia de un ciclo vicioso en que proyectos con recursos ya asignados son los que atraen más recursos; y la ausencia de liderazgo regional y de soluciones colectivas. Dificultades intensificadas con las crecientes divergencias políticas entre los gobiernos de la región. El resultado fue la continuidad de un bajo volumen de inversiones regionales en infraestructura.

Así, el aporte de recursos financieros sigue siendo un desafío para los proyectos de integración regional. La comparación entre las dos carteras prioritarias analizadas en ese trabajo, AIC y API, revela que solo la mitad del monto estimado se encontraba en ejecución o en etapa de conclusión. Eso es poco, teniendo presente que se tratan de proyectos prioritarios cuyo criterio de selección es la disposición de recursos asignados en el presupuesto para el inicio de la ejecución de los mismos. Es decir, incluso los proyectos con fuentes de recursos ya identificadas encuentran dificultad de financiación.

En relación a la inversión pública, episodios de crisis económicas, muy típicas de la región, pueden llevar a la interrupción de las obras. Además, la ejecución de proyectos financiados por presupuestos nacionales crea dificultades legales para proyectos transnacionales, debido al contraste entre distintos ordenamientos jurídicos para los procesos de contratación y adquisición. De hecho, el alta de los precios de las commodities proporcionó el aumento de los ingresos públicos, garantizando cierta autonomía para los gobiernos nacionales. Sin embargo, esa realidad, contemporáneamente, se agotó.

La ausencia de un mecanismo tipo unbundling entre financiación y ejecución del proyecto lleva a un círculo vicioso en el que los proyectos que tienen los recursos asignados en el presupuesto nacional presentan más oportunidades para captar nuevas fuentes de financiación que los proyectos sin recursos previamente asignados. Por lo tanto, los proyectos efectivamente implementados no serán necesariamente los que disponen de mayor alcance regional, sino los que cuentan con presupuesto público. Así, disponer de recursos asignados por el presupuesto es un criterio para que el proyecto pase a formar parte de la agenda de prioridades. 
$\mathrm{Ni}$ el sector privado ni las instituciones multilaterales ejercieron la función de mejorar la calidad crediticia de la cartera de los proyectos para aumentar la captación de nuevos financiamientos. La ausencia de apoyo de las instituciones más fuertes de la región (BID y Banco Mundial) redujeron la capacidad crediticia de la cartera para atracción de inversiones extranjeras. Además de las dificultades regulatorias y políticas, la crisis económica mundial de 2008 también pudo haber contribuido al menor volumen de inversión en regiones pobres y a la desaceleración de las inversiones privadas.

Otro problema institucional destacado fue la falta de un órgano de gestión y de liderazgo. Las visiones de Brasil y Venezuela fueron muy divergentes, no favoreciendo el establecimiento de propuestas consensuadas. Brasil defendía la utilización de las estructuras de financiamiento ya existentes, incluyendo el BNDES, y dejó claro que no iba a profundizar su papel y responsabilidad como paymaster regional.

Las dificultades contextuales en alcanzar un consenso sobre las directrices de integración económica en el ámbito de la Unasur contribuyeron a poner en jaque los delineamientos futuros de la organización. Además, desde 2011, los gobiernos de Colombia, Perú, Chile y México han lanzado una nueva iniciativa, la Alianza del Pacífico, defendiendo el regionalismo abierto y la búsqueda de inversión y financiamiento externos a la región. Esta división se ha vuelto relevante en América del Sur y está evidente en la actual crisis de la Unasur, cuyo fracaso es, sin duda, prejudicial para la inserción económica internacional de la región.

\section{REFERENCIAS}

BULMER-THOMAS, V. The economic history of Latin America since independence. 2. ed. New York: Cambridge University Press, 2003.

CÉSPEDES, S. P.; AGOSTINIS, G. Constructing regionalism in South America: the cases of transport infrastructure and energy within Unasur. Fiesole: EUI, 2014. p. 16-28. (Working Paper, n. 2014/73).

COSTA, C. E. L.; GONZALEZ, M. J. F. Infraestrutura e integração regional: a experiência da IIRSA na América do Sul. Boletim de Economia e Política Internacional, n. 18, set.-dez. 2014.

Infraestrutura e integraçáo regional: uma avaliação da Iniciativa para Integração da Infraestrutura Regional da América do Sul. Rio de Janeiro: Ipea, 2015. (Texto para Discussáo, n. 2060).

COUTO, L. F. Desenvolvimento, integraçáo e assimetrias: caminhos e descaminhos da aproximação regional na América do Sul. 2012. Tese (Doutorado) - Universidade de Brasília, Brasília, 2012. Disponible en: <https:// repositorio.unb.br/handle/10482/11226>. 
FURTADO, C. A economia latino-americana: formação histórica e problemas contemporâneos. São Paulo: Companhia Editora Nacional, 1970.

GRUPO ANALISTAS - ANALISTAS FINANCIEROS INTERNACIONALES. Financiación de proyectos transnacionales de infraestructuras en América del Sur (iniciativa IIRSA). 19. ed. Madrid: BID; IIRSA, 2002. Disponível em: $<$ http://www.iirsa.org/admin_iirsa_web/Uploads/Documents/fid_financiamiento_ proyectos_transnacionales_de_infraestructura.pdf>.

IIRSA - INTEGRACIÓN DE LA INFRAESTRUCTURA REGIONAL SURAMERICANA. Reunión de ministros de Transporte, Telecomunicaciones y Energía de América del Sur: plan de acción para la Integración de la Infraestructura Regional em América del Sur. Montevideo: BID; CAF; FonPlata, 2000. Disponible en: <http://www.iirsa.org/admin_iirsa_web/Uploads/ Documents/Plan\%20de\%20Acci\%C3\%B3n\%20Montevideo\%20final.pdf>.

IV Reunión del Comité de Dirección Ejecutiva. Lima, 23 y 24 nov. 2004.

Agenda de implementación consensuada 2005-2010: informe de evaluación. Buenos Aires: IIRSA, 2010. Disponível em: <http://www.iirsa.org/admin_ iirsa_web/Uploads/Documents/aic_2005_2010_informe_de_evaluacion.pdfs.

IIRSA 10 años después: sus logros y desafíos. Buenos Aires: BID-Intal, 2011. Disponible en: <http://www.iirsa.org/admin_iirsa_web/ Uploads/Documents/lb_iirsa_10_a\%CC3\%B1os_sus_logros_y_desafios.pdf >.

Plan de Acción Estratégico 2012-2022 y Plan de Trabajo 2012. Buenos Aires: IIRSA, 2012. Disponível em: <http://www.iirsa.org/admin_iirsa_ web/Uploads/Documents/oe_api_seminario_difusion_pae_y_pt2012.pdf>.

Cartera de proyectos 2017. Buenos Aires: IIRSA, 2017a. Disponível em: <http://www.iirsa.org/admin_iirsa_web/Uploads/Documents/CARTERA_ DIGITAL.pdf $>$.

Plan de Acción Estratégico 2012-2022: ajustado a 2017. Buenos Aires: IIRSA; Cosiplan, 2017b. Disponível em: <http://www.iirsa.org/admin_ iirsa_web/Uploads/Documents/PAE_digital.pdf>.

MOREIRA, M. M. IIRSA economic fundamentals. [s.l.]: ITD/INT; IDB, 2006. Disponible en: <https://publications.iadb.org/publications/english/ document/IIRSA-Economic-Fundamentals.pdf>. Acceso en: ene. 2020.

PERROTI, D. E.; SÁNCHEZ, R. J. La brecha de infraestructura en América Latina y Caribe. Santiago: Cepal, 2011. (Serie Recursos Naturales y Infraestructura, n. 153). Disponível em: <https://repositorio.cepal.org/bitstream/ handle/11362/6357/S110095.pdf?sequence $=1>$. 
PREBISCH, R. O desenvolvimento económico da América Latina e alguns de seus problemas principais. Boletín Económico de América Latina, v. 7, n. 1, 1962. Disponível em: <https://repositorio.cepal.org/bitstream/handle/11362/1611/003. pdf?sequence $=1>$.

RIGGIROZZI, P.; TUSSIE, D. The rise of post-hegemonic regionalism in Latin America. In: . (Ed.). The rise of post-hegemonic regionalism: the case in Latin America. London: Springer, 2012.

SANAHUJA, J. A. Regionalismo post-liberal y multilateralismo en Sudamérica: el caso de UNASUR. In: SERBIN, A.; MARTÍNEZ, L.; ROMANZINI JÚNIOR, H. (Coord.). El regionalismo "post-liberal" en América Latina y el Caribe: nuevos actores, nuevos temas, nuevos desafíos - Anuario de la Integración Regional de América Latina y el Caribe 2012. Buenos Aires: Cries, 2012. v. 9. Disponível em: <https://sites. usp.br/prolam/wp-content/uploads/sites/35/2019/05/CCD23_2012-Anuario.pdfs.

SANTOS, F. L. B. dos. Unasul à luz de um contraste: as relaçóes com Venezuela e Colômbia. Austral: Revista Brasileira de Estratégia e Relaçôes Internacionais, v. 5, n. 10, p. 240-263, jul.-dez. 2016.

UNASUL - UNIÁO DENAÇÓES SUL-AMERICANAS. Tratado Constitutivo da Uniáo de Naçóes Sul-Americanas. Brasília: Unasur, 2008.

WEGNER, R. C. Integração e desenvolvimento econômico: estratégias de financiamento do investimento de infraestrutura sul-americana. Economia e Sociedade, v. 27, n. 3, p. 909-938, 2018.

\section{BIBLIOGRAFÍA COMPLEMENTARIA}

CALIXTRE, A. B.; BARROS, P. S. O Banco do Sul e o Brasil na agenda da nova arquitetura financeira regional. Boletim de Economia e Política Internacional, n. 3, jul.-set. 2010.

COSIPLAN - CONSEJO SURAMERICANO DE INFRAESTRUTURA Y PLANEAMIENTO. Propuesta para la creación del Grupo de Trabajo sobre Mecanismos de Financiamento y Garantías. [s.l.]: Cosiplan, 2011. Disponible en: <http://www.iirsa.org/admin_iirsa_web/Uploads/Documents/ rc_brasilia11_5_financiamiento_garantias.pdf>.

. Avanços do Grupo de Trabalho sobre Mecanismos de Financiamento

e Garantías: reuniáo do comitê coordenador. Montevideo: Cosiplan, 26 jun. 2013. 12 slides.

IV Reuniáo Ordinária do Conselho de Ministros: financiamento dos projetos de infraestrutura de Integraçáo (reflexôes a partir do GT de financiamento e garantias). Santiago: Cosiplan, 29 nov. 2013. 12 slides. 
Grupo de Trabalho sobre Mecanismos de Financiamento e Garantias: relatório da IV reuniāo. Rio de Janeiro: Cosiplan, 2014.

Cosiplan, 2017.

Agenda de Proyectos Prioritários de Integración 2017. Buenos Aires:

DEOS, S. S. de; MENDONÇA, A. R. R. de; WEGNER, R. C. Cooperação financeira no Mercosul e o financiamento do investimento. Nova Economia, v. 23, n. 1, p. 155-184, 2013.

IIRSA - INTEGRACIÓN DE LA INFRAESTRUCTURA REGIONAL SURAMERICANA. GTE de financiamiento: estudios realizados. [s.l.]: IIRSA, oct. 2004.

VI Reunión del Comité de Dirección Ejecutiva: proceso sectorial instrumentos de financiamiento. Buenos Aires: IIRSA, 2004.

Agenda de Proyectos Prioritarios de Integración. Buenos Aires: IIRSA, 2011. Disponible en: <http://www.iirsa.org/admin_iirsa_web/Uploads/ Documents/api_agenda_proyectos.pdf>.

MARIANO, M. P. O papel do Brasil na integração da infraestrutura da América do Sul: limites institucionais e possibilidades de mudança. In: DESIDERÁ $\mathrm{NETO}, \mathrm{W}$. A. O Brasil e as novas dimensóes da integraçáo regional. Rio de Janeiro: Ipea, 2014.

MEDEIROS, M. de A.; TEIXEIRA JUNIOR, A. W. M.; REIS, E. G. dos. Cooperação para autonomia? Explicando o paradoxo da política externa brasileira para a Unasul. Revista de Sociologia e Política, v. 25, n. 61, p. 97-123, mar. 2017.

PADULA, R. Da IIRSA ao Cosiplan da Unasul: a integração de infraestrutura na América do Sul nos anos 2000 e suas perspectivas de mudança. In: DESIDERÁ NETO, W. A. (Org.). O Brasil e as novas dimensóes da integraçáo regional. Rio de Janeiro: Ipea, 2014.

RUIZ, J. B. Autonomía y desarrollo en el pensamiento latinoamericano. In:___ (Ed.) Integración latinoamericana y caribeńa: política y economía. Madrid: FCE, 2012. 
